\title{
ATDV: An Image Transforming System
}

\author{
Paula Farago ${ }^{1}$, Ligia Barros ${ }^{1}$, Gerson Cunha ${ }^{2}$, Luiz Landau ${ }^{2}$, \\ and Rosa Maria Costa ${ }^{3}$ \\ ${ }^{1}$ Universidade Federal do Rio de Janeiro - UFRJ \\ Instituto de Matemática - Dept de Ciência da Computação \\ Centro de Tecnologia - Bl. C \\ Rio De Janeiro - Rj - Brasil \\ farago@lamce.ufrj.br, ligia@nce.ufrj.br \\ ${ }^{2}$ Universidade Federal do Rio de Janeiro - UFRJ \\ COPPE - Programa de Eng. Civil \\ Caixa Postal 68552 \\ CEP 21949-900 -Rio de Janeiro - RJ - Brasil \\ \{gerson, landau\} @lamce.ufrj.br \\ ${ }^{3}$ Universidade do Estado do Rio de Janeiro - UERJ \\ IME - Dept. de Informática e Ciência da Computação \\ Rua São Francisco Xavier 524- $6^{\circ} \mathrm{B}$ \\ CEP 20550-013 - Rio de Janeiro - RJ - Brasil \\ rcosta@ime.uerj.br
}

\begin{abstract}
Some macular pathology produces degeneration, which causes a perception loss in specific areas of the visual field. In this case, people visualize "blind spots" that hide part of the images captured by the impaired eye.

The goal of this paper is to present an image distortion system that explores the Virtual Reality technology potential. To implement it we developed a system that is able to obtain the data generated by the visual field exam, and decode it. A camera captured the real images and the Convex Hull algorithm and Isoperimetric Mapping were used to identify the spots limits generating a divergent distortion in the images situated inside of the blind spot.

The modified images can be visualized in a Virtual Reality head mounted display with eye tracking. This system offers opportunities to people with this visual problem to get a general perception of the covered area, reducing the visual loss.
\end{abstract}

\section{Introduction}

Nowadays, the sense of vision is one of the most required and necessary for humans because we have a wide range of visual illustrated information that is presented on the Internet, on television, in cinema, etc.

Some people that had toxoplasmosis, glaucoma or senile macular degeneration can get impairments causing an area of diminished vision within the visual field. In general, they see a "blind spot", denominated scotoma, which continually hides some parts of the central and/or peripheral viewing area. The scotoma position is fixed, 
independent of the vision focus or angle. In the majority of cases, the person can have a perception of the general scene, because our eyes interchange central and peripheral vision all the time, but have great difficulty in visualizing objects in movement.

The goal of this paper is, therefore, to present the ATDV (Apoio Tecnológico aos Deficientes Visuais - Technological Support for Visual Impairments) system that aims at temporarily supporting the vision of a person with this pathology. We assume that there is only one scotoma in each eye and we will treat the worst. This system uses Virtual Reality and Augmented Reality techniques and will generate a deformation in the captured images. The objects that are hidden will be seen on the outside limits of the spot. It permits them to have the general perception of the visualized image.

The visual field (VF) exam measures and defines the spot extension of each user. A micro camera captures the mobile images (streams) and transforms them according to the VF exam results. An Isoperimetric Mapping and a Convex Hull algorithm are applied to the VF data. A specific programming language transforms the images that are projected in real time, by a server, on a Head Mounted Display (HMD) connected to a PC or handheld computer.

In the following sections we will present the basic characteristics of Virtual Reality technology, and some medical applications. Next, the ATDV system is explained: the main concepts of the visual field exam, the module that transforms the images, and the Isoperimetric Mapping technique. An example illustrates the algorithm application. In the Conclusions we summarize and draw final remarks.

\section{Virtual Reality Applications}

Virtual Reality (VR) includes advanced technologies of interface, immersing the user in environments that can be actively interacted with and explored [1]. Moreover, the user can accomplish navigation and interaction in a three-dimension synthetic environment generated by computer using multi-sensory channels. In this case, diverse stimuli can be transmitted by specific devices and perceived by one or more user senses.

There are some devices to reproduce generated images from the head-and-eye movements. The first is the Head Mounted Display (HMD), which provokes an immersion in the user.

Some kinds of VR simulations, such as Augmented Reality (AR), have specific classifications. Augmented Reality attempts to superimpose graphics over a real environment in real-time, and also changes those graphics to accommodate a user's head-and-eye movements. Therefore, AR supplements reality, rather than completely replacing it. Virtual Reality and Augmented Reality have a wide scope of application domains like medicine [2], entertainment [3], design [4], robotics and telerobotics [5].

These technologies are being broadly applied in the medical area. Much research has tested developed and tested virtual environments for treating a wide variety of mental and physical disorders [6], [7], [8], [9].

In the specific literature, there are many works describing image transformations. However, they do not explain in details the main technologies being used. In 1986 [10], NASA engineers proposed some ideas to reduce vision loss caused by different kinds of visual deficits. They developed a simple approach exploring a bi-prism 
arrangement in which text is viewed through two prisms placed base to base. Dagnellie [11] describes some vision rehabilitation projects, using intraocular prosthesis and mentions an application for people with scotoma. Starner [12] remapped images that are presented in a wearable computer with a head-mounted display. He uses a simple 2D 'hyper fisheye' coordinate transformation to magnify texts.

The ATDV presents some differences from these systems. The aim of the software introduced in this article is to provide an integrated platform for the visualization of transformed images in an integrated way. The patient can put the diskette with the results of his VF exam (made previously at doctor's office) in the library's PC, the home's PC or a handheld portable; a program decodes these data, generating the inputs to the Isoparametric module. In such a manner, the patient can put the HMD with camera and eye tracking attached and read or see with less difficulty in real time. The system uses the mesh transformation points and translates them dynamically, producing altered images in real-time.

Next, we present the ATDV system that is composed by modules which generates a personalized image transformation.

\section{ATDV - Technological Supporting for Visual Impairments}

The ATDV system integrates different input-output devices, a micro camera, a video server, an HMD with eye tracking and a Handheld [18], or a Personal Computer.

The ATDV system is considered the Central Module, and is responsible for processing data from the VF exam and for generating the transformed images, as presented in Figure 1.

The Central Module is divided in three stages: "Decoder", "VRML generator" and "Isoperimetric Mapping". All these stages compose a single program implemented in $\mathrm{C}++$ language, and will be described next.

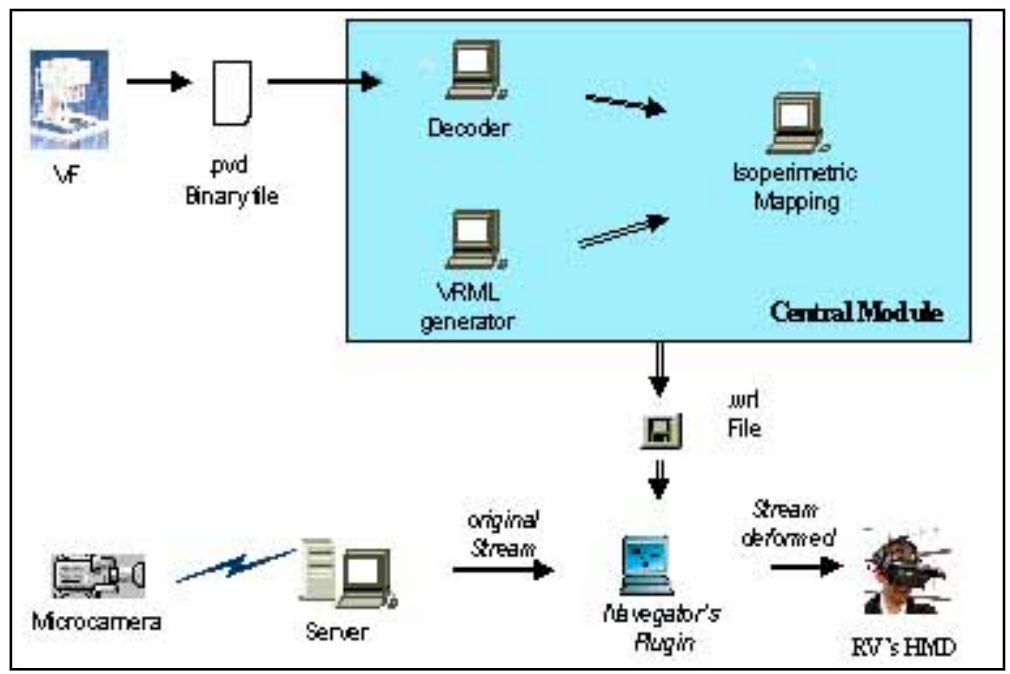

Fig. 1. ATDV system structure 


\subsection{Decoder}

Some diseases such as Toxoplasmosis, Syphilis and Disease of Harada [13] can damage the external and internal part of the Ocular Globe and the optic nerve. This pathological process decreases the visual acuity and may cause lesions denominated scotomas. In general, it is seen as a "black spot" over the visualized images and generates a severe visual field loss.

The scotoma can be evaluated, among other manners, through a visual field exam. It determines the monocular field of view of a person, identifying vision and damaged areas.

The VF exam, from Octopus [17], is made separately on the right and on the left eye. The results are represented by graphics, which include only the $30^{\circ} / 120^{\circ}$ central angle of the total vision field. Various kinds of computerized exam graphics exist, and the Gray Tone Scale, as seen in Figures 2A and 2B, is an example of it. The areas of the same visual perception are shown with the same shade in the gray scale. The area of low visual acuity is the darkest, and the blind refers to the area totally blind, the scotoma.

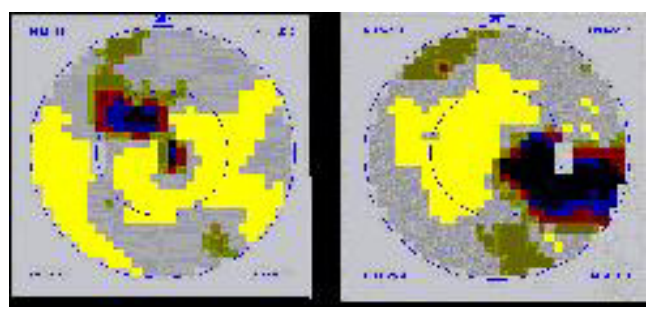

Fig. 2A and 2B. Graphics of Gray tone scale corresponding a $30^{\circ} / 120^{\circ}$ central angle (respectively, left eye and right eye)

The computerized exam generates a binary file (with an .PVD extension) where the relative data is registered, informing the areas with perfect vision and those containing the scotomas. The VF data can only be read by the PeriTrend software, so we had to decode it. Next, these data will be transformed through the VRML Generator and the Isoperimetric Mapping modules.

\subsection{VRML Generator}

The Virtual Reality Modeling Language (VRML) offers some facilities to manipulate the polygon angles trough nodes (Coordinate and Texture Coordinate). In this system, the VRML generator constructs two equal reticulated screens overlapped with n-polygons. The only difference is that the first one will use the Texture Coordinate node to reticulate the texture and the other will use the Coordinate node to serve as a mirror-base flat. Both can be resized to be adapted on the HMD screen by VRML (Navegation Info \{type "Walk" \}).

The captured images are associated to the Coordinate mesh, and the reticulate angles are warped according to the scotoma dimensions by the Isoperimetric Mapping 
techniques. In this way, the information that is inside the scotoma area can be mapped away from the blind retinal area (Figures 6A and 7A).

\subsection{Isoperimetric Mapping and Convex Hull}

The Isoperimetric Mapping in mesh generation was first described by Zienkiewicz and Philips[14]. This technique causes a distortion in images, and uses polynomial interpolation functions to promote a mapping between Cartesian and curvilinear coordinates.

Consider a particular parabolic quadrilateral shape, presented in Figure 3, with eight nodes, in which associated coordinates $\mathrm{X}, \mathrm{Y}$ (and Z) are well defined. In this case, $X=\sum N_{i} x_{i}, Y=\sum N_{i} y_{i}$ and $Z=N_{i} z_{i}$, where $I=1 \ldots 8$. Each $N_{i}$ is defined in terms of a curvilinear coordinates system $\xi$ and $\eta$, that have values between 1 and -1 at the opposed sides.

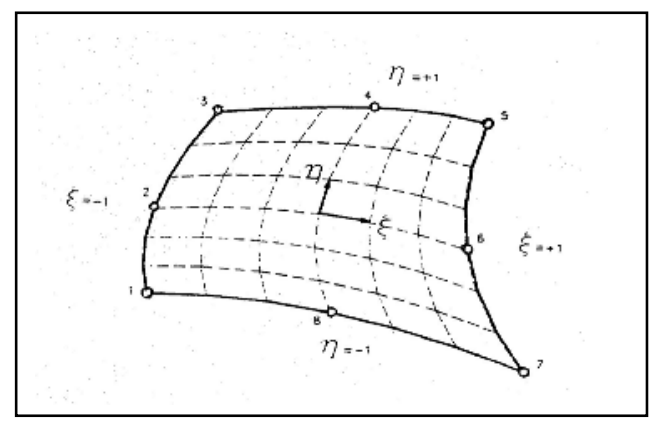

Fig. 3. Parabolic Quadrilateral

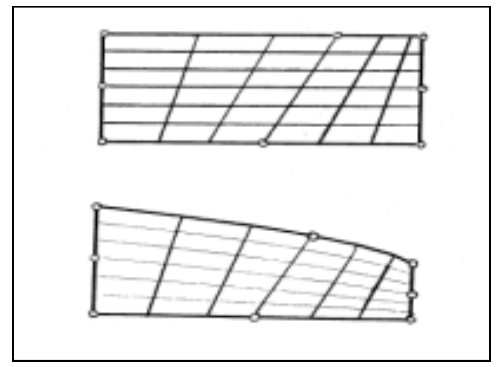

Fig. 4. Mesh transformation

It is used to map the scotoma shape, as shown in Figure 4.

Since the scotoma has a very irregular boundary we have to apply a Convex Hull Algorithm to adjust the transformed mesh to its limits. We used the Graham's scan algorithm [15] and the vectorial product.

To do this, we use the points from the binary .PDV file. Figure 5 presents the adopted technique. 

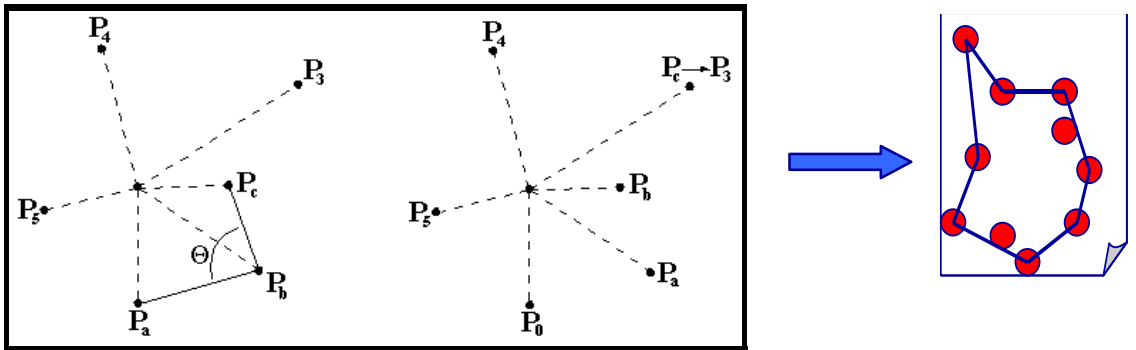

Fig. 5. The algorithm eliminates and translates some points according to scotoma limits. When $\mathrm{Pb}=\mathrm{P} 0$, the process stops, and only the points around the scotoma limits remain

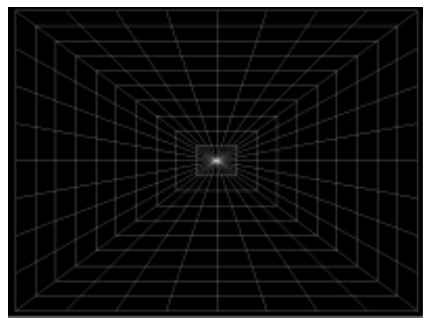

A

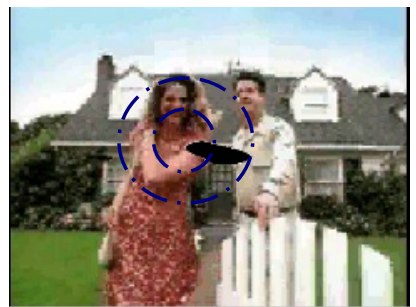

B

Fig. 6. Original coordinate mesh (A) and original texture (B)

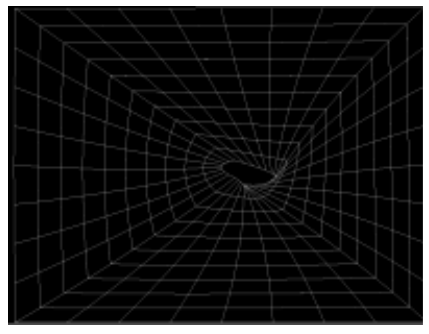

$\mathrm{C}$

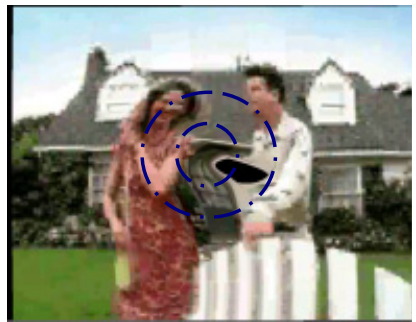

$\mathrm{D}$

Fig. 7. Coordinate mesh (C) and texture (D) deformed by the ATDV system

The information generated by the Central Module are transformed in a .WFL extension file, that keep the mesh that will be used to transform the images that are captured by a micro camera coupled on a head-mounted display (HMD). The Video Server processes these streams, in real time, according to this .WFL file. It distributes these images to the HMD, in unicast model, using a VRML plug in.

An example of this system can be seen in Figures 6 and 7, which present a distortion based on the $30^{\circ} / 120^{\circ}$ center right eye scotoma, showed in Figure 2 . As a result, the objects in the warped area will look "stretched" to the outside border of the scotoma 
This system has preliminarily been tested with only two people. The initial results show that it is useful to read movie captions and watch some games on television. As mentioned in the NASA projects [10] it seems that patients must have time to become accustomed to the devices.

\section{Conclusions}

This article presents an image distortion system that offers new opportunities to people with diminished vision caused by scotomas. The users of this system are persons that have a visual impairment that causes a vision loss in the central field of view, specifically, with only one scotoma per eye.

The system integrates the Isoperimetric and Convex Hull techniques, obtaining a deformed stream that can be visualized in real time in a Virtual Reality device.

We consider that this approach has important technical implications for the development of this kind of Augmented Reality applications.

To spread the use of VR in this domain, we are verifying the possibility of simultaneous images projection to both eyes. This will demand a larger processing capacity in order not to delay the modified images generation. Future experiments should be designed in an attempt to overcome this obstacle.

Lastly, we would like to emphasize that the advances in wearable computers and monocular screens will open new possibilities to the use of this kind of system in this specific domain.

\section{References}

1. Burdea, G., Coiffet, P.(ed): Virtual Reality Technology, $2^{\text {nd }}$ edition, Wiley-Interscience, (2003).

2. Barfield, W. , Caudell, T.: Basic Concepts in Wearable Computers and Augmented Reality, In: Fundamentals of Wearable Computers and Augmented Reality, W. Barfield, T. Caudell (eds.), Mahwah, New Jersey, (2001), 3-26.

3. Stapleton, C., Hughes, C., Moshell, M., Micikevicius, P., Altman, M.: Applying Mixed Reality to Entertainment, In:Computer, 35(12), (2002), 122-124.

4. Hoff, W., Vincent, T.: Analysis of Head Pose Accuracy in Augmented Reality, In: IEEE Trans. Visualization and Computer Graphics, 6(4), (2000).

5. Wheeler, A., Brujic-Okretic, V., Hitchin, L., Parker, G.: Augmented Reality Enhancements for the Teleoperation of Remote Mobile Robots, In: Second International Workshop VRMECH'2001, Brussels, (2001).

6. Costa R.M.; Carvalho, L. A.: The Acceptance of Virtual Reality Devices for Cognitive Rehabilitation: a report of positive results with schizophrenia, In: Computer Methods and Programs in Biomedicine, 73, , (2004), 173-182.

7. Matheis, R.; Schulteis, M., Rizzo, A.:Learning and Memory in a virtual office environment, In:Proceedings of Second International Workshop on Virtual Rehabilitation, (2003), 48-54.

8. Broeren, J., Lundberg, M., Molén, T., Samuelsson, C., Bellner, A., Rydmak, M.:Virtual Reality and Hapitcs as an assessment tool for patients with visuospatial neglect: a preliminary study, In:Proceedings of Second International Workshop on Virtual Rehabilitation, (2003), 27-32. 
9. Niniss, H.; Nadif, A.: Simulation of the behavior of a Powered Wheelchair using Virtual Reality, In: $3^{\text {rd }}$ International Conference on Disability, Virtual Reality and Associated Technologies, (2000), 9-14.

10. NASA: Low Vision Tests and devices, In: www.ski.org/rerc/5yrReport/General/V.html. Visited on 2004.

11. Dagnellie, G.: Virtual Technologies aid tin restoring sight to the blind, In: Communication Through virtual technology: Identity, Community and Technology in the Internet age, G. Riva and F. Davide (eds), IOS Press, (2003), 247-272.

12. Staner, T., Mann, S., Rhodes, B., Levine, J.: Augmented Reality Through Wearable Computing, In Presence, 6(4), (1997), 386-389.

13. Miller, S.: Enfermidades dos olhos e Afecções da retina (Eye Illnesses and Retina Diseases). Ed. Artes Médicas, (1991), 247-269 (in Portuguese).

14. Haber, C.: A General two-dimensional, graphical finite element processor utilizing discrete transfinite mappings. In: International Journal Numerical Methods in Engineering, 17, (1981), $1015-1044$.

15. 15.Riot. In: http://riot.ieor.berkeley.edu/riot/Applications/ConvexHull/CHDetails.html. Visited on 2003.

16. IIS: Inside Information Systems, In: http://www.iis.com.br. Visited on 2001.

17. Octopus. In: http://www.octopus.ch/. Visited on 2005.

18. Poma, In: http://www.xybernaut.com/Solutions/product/downloads/XYBpoma.pdf. Visited on 2005. 